\title{
Numerical Solution of Two-Dimensional Linear Fuzzy Fredholm Integral Equations by the Fuzzy Lagrange Interpolation
}

\author{
H. Nouriani (ID) and R. Ezzati (ID \\ Department of Mathematics, Karaj Branch, Islamic Azad University, Karaj, Iran \\ Correspondence should be addressed to R. Ezzati; ezati@kiau.ac.ir
}

Received 26 October 2017; Accepted 19 May 2018; Published 2 December 2018

Academic Editor: Oscar Castillo

Copyright (C) 2018 H. Nouriani and R. Ezzati. This is an open access article distributed under the Creative Commons Attribution License, which permits unrestricted use, distribution, and reproduction in any medium, provided the original work is properly cited.

In this study, at first, we propose a new approach based on the two-dimensional fuzzy Lagrange interpolation and iterative method to approximate the solution of two-dimensional linear fuzzy Fredholm integral equation (2DLFFIE). Then, we prove convergence analysis and numerical stability analysis for the proposed numerical algorithm by two theorems. Finally, by some examples, we show the efficiency of the proposed method.

\section{Introduction}

Recently many authors proposed various numerical methods for solving one-dimensional fuzzy integral equations [19]. Also, two-dimensional fuzzy integral equations have been noticed by a lot of researchers because of their broad applications in engineering sciences. Some of the most important papers in this area are trapezoidal quadrature rule and iterative method [10-12], triangular functions [13], quadrature iterative [14], Bernstein polynomials [15], collocation fuzzy wavelet like operator [16], homotopy analysis method (HAM) [17], open fuzzy cubature rule [18], kernel iterative method [19], modified homotopy pertubation [20], block-pulse functions [21], optimal fuzzy quadrature formula [22], and finally, iterative method and fuzzy bivariate block-pulse functions [23]. Also, some researchers have solved one-dimensional fuzzy Fredholm integral equations by using fuzzy interpolation via iterative method such as: iterative interpolation method [9], Lagrange interpolation based on the extension principle [5], and spline interpolation [7].

As we know interpolation is one of the most substantial and the most applicable methods in numerical analysis. So, in this paper, we want to solve 2DLFFIEs by applying two-dimensional fuzzy Lagrange interpolation and iterative method. First of all an approximate solution of integral by applying Lagrange interpolation and iterative method is provided. Then, convergence analysis and numerical stability analysis of the proposed method in Theorems 11 and 12 are proved.

The paper is organized as follows: Some notations and theorems about the structure of fuzzy sets are reviewed in Section 2. In Section 3, firstly, we introduce twodimensional fuzzy Lagrange interpolation. Also, we propose two-dimensional fuzzy Lagrange interpolation and iterative method for solving 2DLFFIEs. In Section 4, we verify convergence analysis for proposed method. Also, in Section 5, we prove numerical stability analysis for the method. Two numerical examples are presented in Section 6.

\section{Preliminaries}

At first, we review some basic definitions and necessary results about fuzzy set theory.

Definition 1 (see [24]). A fuzzy number is a function $\tilde{f}$ : $\mathbb{R} \longrightarrow[0,1]$ with the following properties:

(1) $\exists x_{0} \in \mathbb{R}$ such that $\tilde{f}\left(x_{0}\right)=1$.

(2) $\tilde{f}(\eta x+(1-\eta) y) \geq \min \{\tilde{f}(x), \tilde{f}(y)\}, \forall x, y \in \mathbb{R}, \forall \eta \in$ $[0,1]$.

(3) $\forall x_{0} \in \mathbb{R}$ and $\forall \epsilon>0, \exists$ neighborhood $U\left(x_{0}\right): \tilde{f}(x) \leq$ $\widetilde{f}\left(x_{0}\right)+\epsilon, \forall x \in U\left(x_{0}\right)$. 
(4) In $\mathbb{R}$, the set $\overline{\operatorname{supp}(\widetilde{f})}$ is compact.

The set of all fuzzy numbers is denoted by $\mathbb{R}_{F}$.

Definition 2 (see [24]). For $\tilde{f} \in \mathbb{R}_{F}$ and $0<\alpha \leq 1$, define $[\tilde{f}]^{0}:=\overline{\{x \in \mathbb{R}: \widetilde{f}(x)>0\}}$ and

$$
[\tilde{f}]^{\alpha}:=\{x \in \mathbb{R}: \tilde{f}(x) \geq \alpha\} .
$$

Then it is well known that, for each $\alpha \in[0,1],[\tilde{f}]^{\alpha}$ is a bounded and closed interval of $\mathbb{R}$. We define uniquely the sum $\tilde{f} \oplus \widetilde{g}$ and the product $\mu \odot \widetilde{f}$ for $\tilde{f}, \widetilde{g} \in \mathbb{R}_{F}$ and $\mu \in \mathbb{R}$ by

$$
\begin{aligned}
& {[\tilde{f} \oplus \tilde{g}]^{\alpha}=[\tilde{f}]^{\alpha}+[\tilde{g}]^{\alpha},} \\
& {[\mu \odot \tilde{f}]^{\alpha}=\mu[\tilde{f}]^{\alpha},}
\end{aligned}
$$

$$
\forall \alpha \in[0,1]
$$

where $[\tilde{f}]^{\alpha}+[\tilde{g}]^{\alpha}$ means the usual addition of two intervals (as subsets of $\mathbb{R}$ ) and $\mu[\tilde{f}]^{\alpha}$ means the usual product between a scalar and a subset of $\mathbb{R}$. Notice $1 \odot \tilde{f}=\tilde{f}$ and it holds $\tilde{f} \oplus \widetilde{g}=$ $\tilde{g} \oplus \tilde{f}, \mu \odot \tilde{f}=\tilde{f} \odot \mu$. If $0 \leq \alpha_{1} \leq \alpha_{2} \leq 1$ then $[\tilde{f}]^{\alpha_{2}} \subseteq[\tilde{f}]^{\alpha_{1}}$. Actually $[\tilde{f}]^{\alpha}=\left[\tilde{f}_{-}^{(\alpha)}, \tilde{f}_{+}^{(\alpha)}\right]$, where $\widetilde{f}_{-}^{(\alpha)} \leq \widetilde{f}_{+}^{(\alpha)}, \widetilde{f}_{-}^{(\alpha)}, \tilde{f}_{+}^{(\alpha)} \in \mathbb{R}$, $\forall \alpha \in[0,1]$. For $\mu>0$ one has $\mu \widetilde{f}_{ \pm}^{(\alpha)}=(\mu \odot \widetilde{f})_{ \pm}^{(\alpha)}$, respectively.

Definition 3 (see [24]). Define $D: \mathbb{R}_{F} \times \mathbb{R}_{F} \longrightarrow \mathbb{R}_{+}$by

$$
\begin{aligned}
D(\tilde{f}, \tilde{g}) & :=\sup _{\alpha \in[0,1]} \max \left\{\left|\tilde{f}_{-}^{(\alpha)}-\tilde{g}_{-}^{(\alpha)}\right|,\left|\tilde{f}_{+}^{(\alpha)}-\tilde{g}_{+}^{(\alpha)}\right|\right\} \\
& =\sup _{\alpha \in[0,1]} \text { Hausdorff distance }\left([\tilde{f}]^{\alpha},[\tilde{g}]^{\alpha}\right),
\end{aligned}
$$

where $[\tilde{g}]^{\alpha}=\left[\widetilde{g}_{-}^{(\alpha)}, \widetilde{g}_{+}^{(\alpha)}\right] ; \tilde{f}, \tilde{g} \in \mathbb{R}_{F}$. Clearly $D$ is a metric on $\mathbb{R}_{F}$. Also $\left(\mathbb{R}_{F}, D\right)$ is a complete metric space, with the following properties [24]:

$$
\begin{aligned}
& D(\tilde{f} \oplus \tilde{h}, \tilde{g} \oplus \tilde{h})=D(\tilde{f}, \tilde{g}), \quad \forall \tilde{f}, \tilde{g}, \tilde{h} \in \mathbb{R}_{F}, \\
& D\left(k^{\prime} \odot \tilde{f}, k^{\prime} \odot \tilde{g}\right)=\left|k^{\prime}\right| D(\tilde{f}, \tilde{g}), \\
& \forall \tilde{f}, \widetilde{g} \in \mathbb{R}_{F}, \forall k^{\prime} \in \mathbb{R}, \\
& D(\tilde{f} \oplus \widetilde{g}, \tilde{h} \oplus \widetilde{e}) \leq D(\widetilde{f}, \widetilde{h})+D(\widetilde{g}, \widetilde{e}), \\
& \forall \tilde{f}, \tilde{g}, \tilde{h}, \widetilde{e} \in \mathbb{R}_{F} .
\end{aligned}
$$

Definition 4 (see [24]). Suppose $\tilde{f}, \tilde{g}: \mathbb{R} \longrightarrow \mathbb{R}_{F}$ be fuzzy number valued functions, then the distance between $f$ and $g$ is defined by

$$
D^{*}(\tilde{f}, \tilde{g}):=\sup _{x \in \mathbb{R}} D(\tilde{f}(x), \widetilde{g}(x)) .
$$

Lemma 5 (see [24]).

(1) If we denote $\widetilde{0}:=\chi_{\{0\}}$, then $\forall \tilde{f} \in \mathbb{R}_{F}, \tilde{f} \oplus \widetilde{0}=\widetilde{0} \oplus \tilde{f}=$ $\widetilde{f}$.
(2) With respect to $\tilde{0}$, none of $\tilde{f} \in \mathbb{R}_{F}, \tilde{f} \neq \tilde{0}$ has opposite in $\mathbb{R}_{F}$.

(3) Let $\alpha, \beta \in \mathbb{R}: \alpha \cdot \beta \geq 0$, and any $\tilde{f} \in \mathbb{R}_{F}$, we have $(\alpha+\beta) \odot \widetilde{f}=\alpha \odot \widetilde{f} \oplus \beta \odot \widetilde{f}$. Notice that, for general $\alpha$, $\beta \in \mathbb{R}$, the above property is false.

(4) For any $\gamma \in \mathbb{R}$ and any $\tilde{f}, \widetilde{g} \in \mathbb{R}_{F}$, we have $\gamma \odot(\tilde{f} \oplus \widetilde{g})=$ $\gamma \odot \tilde{f} \oplus \gamma \odot \tilde{g}$.

(5) For any $\gamma, \eta \in \mathbb{R}$ and any $\tilde{f} \in \mathbb{R}_{F}$, we have $\gamma \odot(\eta \odot \tilde{f})=$ $(\gamma \odot \eta) \odot \tilde{f}$.

If we denote $\|\tilde{f}\|_{F}:=D(\tilde{f}, \tilde{0}), \forall \tilde{f} \in \mathbb{R}_{F}$, then $\|\cdot\|_{F}$ has the properties of a usual norm on $\mathbb{R}_{F}$, i.e.,

$$
\begin{aligned}
\|\tilde{f}\|_{F} & =0 \quad \text { iff } \tilde{f}=\tilde{0}, \\
\|\mu \odot \tilde{f}\|_{F} & =|\mu| \cdot\|\tilde{f}\|_{F}, \\
\|\tilde{f} \oplus \tilde{g}\|_{F} & \leq\|\tilde{f}\|_{F}+\|\tilde{g}\|_{F}, \\
\|\widetilde{f}\|_{F}-\|\tilde{g}\|_{F} & \leq D(\tilde{f}, \tilde{g}) .
\end{aligned}
$$

Notice that $\left(\mathbb{R}_{F}, \oplus, \odot\right)$ is not a linear space over $\mathbb{R}$, and consequently $\left(\mathbb{R}_{F},\|\cdot\|_{F}\right)$ is not a normed space. Here $\sum^{*}$ denotes the fuzzy summation.

Definition 6 (see [24]). A fuzzy valued function $\tilde{f}:[a, b] \longrightarrow$ $\mathbb{R}_{F}$ is said to be continuous at $x_{0} \in[a, b]$, if for each $\epsilon>0$ there exists $\delta>0$ such that $D\left(\widetilde{f}(x), \widetilde{f}\left(x_{0}\right)\right)<\epsilon$, whenever $x \in[a, b]$ and $\left|x-x_{0}\right|<\delta$. We say that $\tilde{f}$ is fuzzy continuous on $[a, b]$ if $\tilde{f}$ is continuous at each $x_{0} \in[a, b]$ and denotes the space of all such functions by $C_{F}[a, b]$.

Definition 7 (see [11]). Suppose that $\tilde{f}:[a, b] \times[c, d] \longrightarrow \mathbb{R}_{f}$ is a bounded mapping. The function $\omega_{[a, b] \times[c, d]}(\tilde{f}, \cdot): \mathbb{R}_{+} \cup$ $\{0\} \longrightarrow \mathbb{R}_{+}$defined by

$$
\begin{gathered}
\omega_{[a, b] \times[c, d]}(\tilde{f}, \delta)=\sup \{D(\tilde{f}(x, y), \tilde{f}(s, t)) ; x, s \\
\left.\in[a, b] ; y, t \in[c, d] ; \sqrt{(x-s)^{2}+(y-t)^{2}} \leq \delta\right\},
\end{gathered}
$$

is called modules of oscillation of $\tilde{f}$ on $[a, b] \times[c, d]$. Also, if $\widetilde{f} \in C_{F}([a, b] \times[c, d])$, then $\omega_{[a, b] \times[c, d]}(\tilde{f}, \delta)$ is called uniform modules of continuity of $\tilde{f}$.

Theorem 8 (see [11]). The following properties hold:

(1) $D(\tilde{f}(x, y), \tilde{f}(s, t)) \leq \omega_{[a, b] \times[c, d]}\left(\tilde{f}, \sqrt{(x-s)^{2}+(y-t)^{2}}\right)$, $\forall x, s \in[a, b], y, t \in[c, d] ;$

(2) $\omega_{[a, b] \times[c, d]}(\tilde{f}, \delta)$ is a nondecreasing mapping in $\delta$;

(3) $\omega_{[a, b] \times[c, d]}(\tilde{f}, 0)=0$;

(4) $\omega_{[a, b] \times[c, d]}\left(\tilde{f}, \delta_{1}+\delta_{2}\right) \leq \omega_{[a, b] \times[c, d]}\left(\tilde{f}, \delta_{1}\right)+\omega_{[a, b] \times[c, d]}(\tilde{f}$, $\left.\delta_{2}\right), \forall \delta_{1}, \delta_{2} \geq 0$; 
(5) $\omega_{[a, b] \times[c, d]}(\tilde{f}, n \delta) \leq n \omega_{[a, b] \times[c, d]}(\tilde{f}, \delta), \forall \delta \geq 0, n \in \mathbb{N}$;

(6) $\omega_{[a, b] \times[c, d]}(\tilde{f}, \mu \delta) \leq(\mu+1) \omega_{[a, b] \times[c, d]}(\tilde{f}, \delta), \forall \mu, \delta \geq 0$.

Theorem 9 (see [11]). If $\tilde{f}$ and $\widetilde{g}$ are Henstock integrable mapping on $[a, b] \times[c, d]$ and if $D(\tilde{f}(s, t), \widetilde{g}(s, t))$ is Lebesgue integrable, then

$$
\begin{aligned}
& D\left((F H) \int_{c}^{d} \int_{a}^{b} \tilde{f}(s, t) d s d t,(F H) \int_{c}^{d} \int_{a}^{b} \widetilde{g}(s, t) d s d t\right) \\
& \quad \leq(L) \int_{c}^{d} \int_{a}^{b} D(\tilde{f}(s, t), \widetilde{g}(s, t)) d s d t .
\end{aligned}
$$

\section{The Main Result}

In this section, first, we introduce two-dimensional fuzzy Lagrange interpolation. Then, we propose two-dimensional fuzzy Lagrange interpolation and iterative method for solving (9).

Consider 2DLFFIE as follows:

$$
\begin{aligned}
& \widetilde{G}(s, t) \\
& =\tilde{g}(s, t) \oplus \mu \\
& \quad \odot(F R) \int_{c}^{d}(F R) \int_{a}^{b} \mathscr{K}(x, y, s, t) \odot \widetilde{G}(x, y) d x d y,
\end{aligned}
$$

where $\mu>0, \mathscr{K}(x, y, s, t)$ is an arbitrary positive function on $[a, b] \times[c, d] \times[a, b] \times[c, d]$ and $\tilde{g}:[a, b] \times[c, d] \longrightarrow \mathbb{R}_{F}$. We assume that $\mathscr{K}$ is continuous, and therefore it is uniformly continuous with respect to $(s, t)$. So, there exists $\mathscr{M}>0$ such that $\mathscr{M}=\max _{s, x \in[a, b], y, t \in[c, d]}|\mathscr{K}(x, y, s, t)|$.

Two-dimensional Lagrange polynomials, $\ell_{i j}(s, t)$, are defined as follows:

$$
\ell_{i j}(s, t)=\ell_{i}(s) \otimes \ell_{j}(t), \quad 0 \leq i, j \leq n,
$$

where $\ell_{i}(s)$ is the Lagrange polynomial and is defined as follows:

$$
\ell_{i}(s)=\prod_{r=0, r \neq i}^{n} \frac{\left(s-s_{r}\right)}{\left(s_{i}-s_{r}\right)}
$$

therefore,

$$
\ell_{i j}\left(s_{r}, t_{r^{\prime}}\right)= \begin{cases}1, & \text { if } i=r, j=r^{\prime} \\ 0, & \text { otherwise. }\end{cases}
$$

So, the two-dimensional interpolation in the Lagrange form is (see [25])

$$
p(x, y)=\sum_{j=0}^{n} \sum_{i=0}^{n} \tilde{f}\left(s_{i}, t_{j}\right) \odot \ell_{i j}(x, y),
$$

where the coefficients $\tilde{f}\left(s_{i}, t_{j}\right)$ are the fuzzy numbers.
Here, we consider the two-dimensional fuzzy Lagrange interpolation in the given points $a=s_{0}<s_{1}<\cdots<s_{n}=b$ and $c=t_{0}<t_{1}<\cdots<t_{n}=d$ such that

$$
\begin{aligned}
& \mathscr{K}(x, y, s, t) \odot \widetilde{G}(x, y) \\
& \quad \approx \sum_{j=0}^{n} \sum_{i=0}^{n} \ell_{i j}(x, y) \odot \mathscr{K}\left(s_{i}, t_{j}, s, t\right) \odot \widetilde{G}\left(s_{i}, t_{j}\right) .
\end{aligned}
$$

Now, we propose a numerical method to solve (9). To do this, we suppose the following iterative procedure to approximate the solution of (9) in point $(s, t)$

$$
\begin{aligned}
\widetilde{u}_{0}(s, t)= & \tilde{g}(s, t), \\
\widetilde{u}_{k}(s, t)= & \tilde{g}(s, t) \oplus \mu \\
& \odot \sum_{j=0}^{n} \sum_{i=0}^{n} C_{i j} \mathscr{K}\left(s_{i}, t_{j}, s, t\right) \odot \widetilde{u}_{k-1}\left(s_{i}, t_{j}\right),
\end{aligned}
$$

where

$$
C_{i j}=\int_{c}^{d} \int_{a}^{b} \ell_{i j}(x, y) d x d y .
$$

In Theorem 10, authors of [11] proved the existence and uniqueness solution of (9) by using the Banach fixed point theorem.

Theorem 10 (see [11]). Let the function $\mathscr{K}(x, y, s, t)$ be continuous and positive for $x, s \in[a, b]$, and $y, t \in[c, d]$, and let $\tilde{g}:[a, b] \times[c, d] \longrightarrow \mathbb{R}_{F}$ be continuous on $[a, b] \times[c, d]$. If $B=\mu M(d-c)(b-a)<1$ then the fuzzy integral equation (9) has a unique solution $\widetilde{G}^{*} \in X$, where

$$
X=\left\{\tilde{g}:[a, b] \times[c, d] \longrightarrow \mathbb{R}_{F} ; \tilde{g} \text { is continous }\right\},
$$

be the space of two-dimensional fuzzy continuous functions with the metric $D^{*}$ and it can be obtained by the following successive approximations method:

$$
\begin{aligned}
& \widetilde{G}_{0}(s, t)=\widetilde{g}(s, t), \\
& \widetilde{G}_{k}(s, t)=\widetilde{g}(s, t) \oplus \mu \odot(F R)
\end{aligned}
$$

$$
\cdot \int_{c}^{d}(F R) \int_{a}^{b} \mathscr{K}(x, y, s, t) \odot \widetilde{G}_{k-1}(x, y) d x d y,
$$

$\forall k \geq 1$.

Moreover, the sequence of successive approximations $\left(\widetilde{G}_{k}\right)_{k \geq 1}$ converges to the solution $\widetilde{G}^{*}$. Furthermore, the following error bound holds:

$$
D^{*}\left(\widetilde{G}^{*}, \widetilde{G}_{k}\right) \leq \frac{B^{k+1}}{1-B} \mathscr{M}_{1}, \quad \forall k \geq 1,
$$

where $\mathscr{M}_{1}=\sup _{s \in[a, b], t \in[c, d]}\|\widetilde{G}(s, t)\|$. 


\section{Convergence Analysis}

In this section, we obtain an error estimate between the exact

$$
+\frac{1}{1-B}\left(\left(1+\frac{2 \widetilde{C}}{(d-c)(b-a)}\right) m_{l}\right) .
$$

solution and the approximate solution of 2DLFFIE (9).

Theorem 11. Under the hypotheses of Theorem 10 and $\mu>0$, the iterative procedure (15) converges to the unique solution of (9), $\widetilde{G}^{*}$, and its error estimate is as follows:

$$
\begin{aligned}
& D^{*}\left(\widetilde{G}^{*}, \widetilde{u}_{k}\right) \\
& \quad \leq \frac{B^{k+1}}{(1-B)} \mathscr{M}_{1}
\end{aligned}
$$

Proof. Clearly, we have

$$
\begin{aligned}
D\left(\widetilde{G}^{*}(s, t), \widetilde{u}_{k}(s, t)\right) \leq & D\left(\widetilde{G}^{*}(s, t), \widetilde{G}_{k}(s, t)\right) \\
& +D\left(\widetilde{G}_{k}(s, t), \widetilde{u}_{k}(s, t)\right) .
\end{aligned}
$$

From (18) and (15), we conclude that

$$
\begin{aligned}
& D\left(\widetilde{G}_{k}(s, t), \widetilde{u}_{k}(s, t)\right)=D\left(\widetilde{g}(s, t) \oplus \mu \odot(F R) \int_{c}^{d}(F R) \int_{a}^{b} \mathscr{K}(x, y, s, t) \odot \widetilde{G}_{k-1}(x, y) d x d y, \widetilde{g}(s, t) \oplus \mu \odot \sum_{j=0}^{n} \sum_{i=0}^{n} C_{i j} \mathscr{K}\left(s_{i}, t_{j}, s, t\right) \odot \widetilde{u}_{k-1}\left(s_{i}, t_{j}\right)\right)=\mu D\left((F R) \int_{c}^{d}(F R)\right. \\
& \left.\cdot \int_{a}^{b} \mathscr{K}(x, y, s, t) \odot \widetilde{G}_{k-1}(x, y) d x d y, \sum_{j=0}^{n} \sum_{i=0}^{n} C_{i j} \mathscr{K}\left(s_{i}, t_{j}, s, t\right) \odot \widetilde{u}_{k-1}\left(s_{i}, t_{j}\right)\right)=\mu D\left(\sum_{j=1}^{n} \sum_{i=1}^{n}(F R) \int_{t_{j-1}}^{t_{j}}(F R) \int_{s_{i-1}}^{s_{i}} \mathscr{K}(x, y, s, t) \odot \widetilde{G}_{k-1}(x, y) d x d y,\right. \\
& \left.\sum_{j=0}^{n} \sum_{i=0}^{n} C_{i j} \mathscr{K}\left(s_{i}, t_{j}, s, t\right) \odot \widetilde{u}_{k-1}\left(s_{i}, t_{j}\right)\right)=\mu D\left(\sum_{j=1}^{n} \sum_{i=1}^{n}(F R) \int_{t_{j-1}}^{t_{j}}(F R) \int_{s_{i-1}}^{s_{i}} \mathscr{K}(x, y, s, t) \odot \widetilde{G}_{k-1}(x, y) d x d y, \sum_{j=1}^{n} \sum_{i=1}^{n}\left(t_{j}-t_{j-1}\right)\left(s_{i}-s_{i-1}\right) \frac{C_{i j}}{\left(t_{j}-t_{j-1}\right)\left(s_{i}-s_{i-1}\right)} \mathscr{K}\left(s_{i}, t_{j}, s, t\right)\right. \\
& \left.\odot \widetilde{u}_{k-1}\left(s_{i}, t_{j}\right) \oplus C_{00} \mathscr{K}\left(s_{0}, t_{0}, s, t\right) \odot \widetilde{u}_{k-1}\left(s_{0}, t_{0}\right)\right) \\
& \leq \mu \sum_{j=1}^{n} \sum_{i=1}^{n} D\left((F R) \int_{t_{j-1}}^{t_{j}}(F R) \int_{s_{i-1}}^{s_{i}} \mathscr{K}(x, y, s, t) \odot \widetilde{G}_{k-1}(x, y) d x d y,(F R) \int_{t_{j-1}}^{t_{j}}(F R) \int_{s_{i-1}}^{s_{i}} \frac{C_{i j}}{\left(t_{j}-t_{j-1}\right)\left(s_{i}-s_{i-1}\right)} \mathscr{K}\left(s_{i}, t_{j}, s, t\right) \odot \widetilde{u}_{k-1}\left(s_{i}, t_{j}\right) d x d y\right) \oplus \mu D\left(C_{00} \mathscr{K}\left(s_{0}, t_{0}, s, t\right)\right. \\
& \left.\odot \tilde{u}_{k-1}\left(s_{0}, t_{0}\right), \widetilde{0}\right) \\
& \leq \mu \sum_{j=1 i=1}^{n} \sum_{i=1}^{n} \int_{t_{j-1}}^{t_{j}} \int_{s_{i-1}}^{s_{i}}\left[D\left(\mathscr{K}(x, y, s, t) \odot \widetilde{G}_{k-1}(x, y), \mathscr{K}(x, y, s, t) \odot \widetilde{u}_{k-1}\left(s_{i}, t_{j}\right)\right)+D\left(\mathscr{K}(x, y, s, t) \odot \widetilde{u}_{k-1}\left(s_{i}, t_{j}\right), \frac{C_{i j}}{\left(t_{j}-t_{j-1}\right)\left(s_{i}-s_{i-1}\right)} \mathscr{K}\left(s_{i}, t_{j}, s, t\right) \odot \widetilde{u}_{k-1}\left(s_{i}, t_{j}\right)\right)\right] d x d y \\
& +\mu D\left(C_{00} \mathscr{K}\left(s_{0}, t_{0}, s, t\right) \odot \widetilde{u}_{k-1}\left(s_{0}, t_{0}\right), \widetilde{0}\right) \\
& \leq \mu \sum_{j=1}^{n} \sum_{i=1}^{n} \int_{t_{j-1}}^{t_{j}} \int_{s_{i-1}}^{s_{i}}\left(|\mathscr{K}(x, y, s, t)| D\left(\widetilde{G}_{k-1}(x, y), \widetilde{u}_{k-1}\left(s_{i}, t_{j}\right)\right)+|\mathscr{K}(x, y, s, t)| D\left(\widetilde{u}_{k-1}\left(s_{i}, t_{j}\right), \tilde{0}\right)+\left|\frac{C_{i j}}{\left(t_{j}-t_{j-1}\right)\left(s_{i}-s_{i-1}\right)} \mathscr{K}\left(s_{i}, t_{j}, s, t\right)\right| D\left(\widetilde{u}_{k-1}\left(s_{i}, t_{j}\right), \widetilde{0}\right)\right) d x d y \\
& +\mu D\left(C_{00} \mathscr{K}\left(s_{0}, t_{0}, s, t\right) \odot \widetilde{u}_{k-1}\left(s_{0}, t_{0}\right), \widetilde{0}\right) .
\end{aligned}
$$

By supposing $m_{k-1}=\sup _{(s, t) \in[a, b] \times[c, d]}\left\|\widetilde{u}_{k-1}(s, t)\right\|$, we get

$$
\begin{aligned}
D & \left(\widetilde{G}_{k}(s, t), \widetilde{u}_{k}(s, t)\right) \\
& \leq \mu \sum_{j=1}^{n} \sum_{i=1}^{n} \int_{t_{j-1}}^{t_{j}} \int_{s_{i-1}}^{s_{i}}\left(\mathscr{M} D\left(\widetilde{G}_{k-1}(x, y), \widetilde{u}_{k-1}\left(s_{i}, t_{j}\right)\right)+\mathscr{M} m_{k-1}+\left|\frac{C_{i j}}{\left(t_{j}-t_{j-1}\right)\left(s_{i}-s_{i-1}\right)} \mathscr{K}\left(s_{i}, t_{j}, s, t\right)\right| m_{k-1}\right) d x d y \\
& +\mu\left|C_{00} \mathscr{K}\left(s_{0}, t_{0}, s, t\right)\right| m_{k-1} .
\end{aligned}
$$

Also, we have

$$
\begin{aligned}
D\left(\widetilde{G}_{k}(s, t), \widetilde{u}_{k}(s, t)\right) \leq & \mu \sum_{j=1}^{n} \sum_{i=1}^{n} \int_{t_{j-1}}^{t_{j}} \int_{s_{i-1}}^{s_{i}}\left(\mathscr{M} D^{*}\left(\widetilde{G}_{k-1}, \widetilde{u}_{k-1}\right)\right) d x d y+\mu \sum_{j=1}^{n} \sum_{i=1}^{n} \int_{t_{j-1}}^{t_{j}} \int_{s_{i-1}}^{s_{i}} \mathscr{M} m_{k-1} d x d y \\
& +\mu \sum_{j=1}^{n} \sum_{i=1}^{n} \int_{t_{j-1}}^{t_{j}} \int_{s_{i-1}}^{s_{i}}\left|\frac{C_{i j}}{\left(t_{j}-t_{j-1}\right)\left(s_{i}-s_{i-1}\right)} \mathscr{K}\left(s_{i}, t_{j}, s, t\right)\right| m_{k-1} d x d y+\mu\left|C_{00} \mathscr{K}\left(s_{0}, t_{0}, s, t\right)\right| m_{k-1} .
\end{aligned}
$$


Therefore,

$$
\begin{aligned}
D\left(\widetilde{G}_{k}(s, t), \widetilde{u}_{k}(s, t)\right) \\
\leq \mu M(d-c)(b-a) D^{*}\left(\widetilde{G}_{k-1}, \widetilde{u}_{k-1}\right) \\
\quad+\mu \mathscr{M}(d-c)(b-a) m_{k-1} \\
\quad+\mu \mathscr{M}(d-c)(b-a) m_{k-1} \frac{\widetilde{C}}{(d-c)(b-a)} \\
\quad+\mu \mathscr{M} \widetilde{C}_{k-1} \\
\leq B D^{*}\left(\widetilde{G}_{k-1}, \widetilde{u}_{k-1}\right)+B m_{k-1} \\
\quad+2 B m_{k-1} \frac{\widetilde{C}}{(d-c)(b-a)} \\
=B D^{*}\left(\widetilde{G}_{k-1}, \widetilde{u}_{k-1}\right) \\
\quad+B m_{k-1}\left(1+\frac{2 \widetilde{C}}{(d-c)(b-a)}\right)
\end{aligned}
$$

where $\widetilde{C}=\max \left|C_{i j}\right|, i, j=0, \cdots, n$. Hence, we conclude that

$$
\begin{gathered}
D^{*}\left(\widetilde{G}_{k-1}, \widetilde{u}_{k-1}\right) \leq B D^{*}\left(\widetilde{G}_{k-2}, \widetilde{u}_{k-2}\right) \\
+B m_{k-2}\left(1+\frac{2 \widetilde{C}}{(d-c)(b-a)}\right) \\
\vdots \\
D^{*}\left(\widetilde{G}_{1}, \widetilde{u}_{1}\right) \leq B D^{*}\left(\widetilde{G}_{0}, \widetilde{u}_{0}\right) \\
+B m_{0}\left(1+\frac{2 \widetilde{C}}{(d-c)(b-a)}\right) .
\end{gathered}
$$

So,

$$
\begin{aligned}
D^{*}\left(\widetilde{G}_{k}, \widetilde{u}_{k}\right) \leq & \left(1+\frac{2 \widetilde{C}}{(d-c)(b-a)}\right) \\
& \cdot\left(B m_{k-1}+B^{2} m_{k-2}+\cdots+B^{k} m_{0}\right) .
\end{aligned}
$$

If $m_{l}=\max \left\{m_{0}, \ldots, m_{k-1}\right\}$, then we obtain

$$
D^{*}\left(\widetilde{G}_{k}, \widetilde{u}_{k}\right)<\frac{1}{1-B}\left(\left(1+\frac{2 \widetilde{C}}{(d-c)(b-a)}\right) m_{l}\right),
$$

therefore

$$
\begin{aligned}
D^{*}\left(\widetilde{G}^{*}, \widetilde{u}\right) \leq & \frac{B^{k+1}}{1-B} M_{1} \\
& +\frac{1}{1-B}\left(\left(1+\frac{2 \widetilde{C}}{(d-c)(b-a)}\right) m_{l}\right) .
\end{aligned}
$$

\section{Numerical Stability Analysis}

To show the numerical stability analysis of the proposed method in previous section, we consider another starting approximation $\widetilde{f}(s, t)=\widetilde{Y}_{0}(s, t)$ such that $\exists \epsilon>0$ for which $D\left(\widetilde{G}_{0}(s, t), \widetilde{Y}_{0}(s, t)\right)<\epsilon, \forall s, t \in[a, b] \times[c, d]$. The obtained sequence of successive approximations is

$$
\begin{aligned}
& \tilde{Y}_{k}(s, t)=\tilde{f}(s, t) \oplus \mu \odot(F R) \\
& \quad \cdot \int_{c}^{d}(F R) \int_{a}^{b} \mathscr{K}(x, y, s, t) \odot \widetilde{Y}_{k-1}(x, y) d x d y,
\end{aligned}
$$

and using the same iterative method, the terms of produced sequence are

$$
\begin{aligned}
\widetilde{v}_{0}(s, t)= & \widetilde{Y}_{0}(s, t)=\tilde{f}(s, t), \\
\widetilde{v}_{k}(s, t)= & \tilde{f}(s, t) \oplus \mu \\
& \odot \sum_{j=0}^{n} \sum_{i=0}^{n} C_{i j} \mathscr{K}\left(s_{i}, t_{j}, s, t\right) \odot \widetilde{v}_{k-1}\left(s_{i}, t_{j}\right) .
\end{aligned}
$$

Theorem 12. The proposed method (15), under the assumptions of Theorem 11, is numerically stable with respect to the choice of the first iteration.

Proof. At first, we obtain that

$$
\begin{aligned}
& D\left(\widetilde{u}_{k}(s, t), \widetilde{v}_{k}(s, t)\right) \\
& \leq D\left(\widetilde{u}_{k}(s, t), \widetilde{G}_{k}(s, t)\right)+D\left(\widetilde{G}_{k}(s, t), \widetilde{Y}_{k}(s, t)\right) \\
&+D\left(\widetilde{Y}_{k}(s, t), \widetilde{v}_{k}(s, t)\right) \\
& \leq \frac{1}{1-B}\left(\left(1+\frac{2 \widetilde{C}}{(d-c)(b-a)}\right) m_{l}\right) \\
&+D\left(\widetilde{G}_{k}(s, t), \widetilde{Y}_{k}(s, t)\right) \\
&+\frac{1}{1-B}\left(\left(1+\frac{2 \widetilde{C}}{(d-c)(b-a)}\right) m_{l}\right) .
\end{aligned}
$$

However,

$$
D\left(\widetilde{G}_{k}(s, t), \widetilde{Y}_{k}(s, t)\right)=D(\widetilde{g}(s, t) \oplus \mu \odot(F R)
$$

$$
\begin{aligned}
& \cdot \int_{c}^{d}(F R) \\
& \cdot \int_{a}^{b} \mathscr{K}(x, y, s, t) \odot \widetilde{G}_{k-1}(x, y) d x d y, \tilde{f}(s, t) \oplus \mu
\end{aligned}
$$$$
\odot(F R) \int_{c}^{d}(F R) \int_{a}^{b} \mathscr{K}(x, y, s, t)
$$$$
\left.\odot \widetilde{Y}_{k-1}(x, y) d x d y\right) \leq D(\widetilde{g}(s, t), \tilde{f}(s, t))
$$$$
+\mu D\left((F R) \int_{c}^{d}(F R) \int_{a}^{b} \mathscr{K}(x, y, s, t)\right.
$$ 
TABLE 1: The absolute errors on the level sets with $n=2, k=5$ for Example 13 by using the proposed method $(s, t)=(0.5,0.5)$.

\begin{tabular}{lcc}
\hline$r$ level & $\underline{e}^{r}=\left|\underline{\underline{G}}^{*}(s, t, r)-\underline{\underline{u}}_{k}(s, t, r)\right|$ & $\bar{e}^{r}=\left|\overline{\widetilde{G}}^{*}(s, t, r)-\overline{\tilde{u}}_{k}(s, t, r)\right|$ \\
\hline 0.0 & 0. & $1.23485 e-5$ \\
0.1 & $6.66819 e-7$ & $1.141 e-5$ \\
0.2 & $1.3501 e-6$ & $1.05703 e-5$ \\
0.3 & $2.04985 e-6$ & $9.82941 e-6$ \\
0.4 & $2.76606 e-6$ & $9.18729 e-6$ \\
0.5 & $3.49874 e-6$ & $8.64395 e-6$ \\
0.6 & $4.24788 e-6$ & $8.19941 e-6$ \\
0.7 & $5.01349 e-6$ & $7.85365 e-6$ \\
0.8 & $5.79556 e-6$ & $7.60668 e-6$ \\
0.9 & $6.5941 e-6$ & $7.45849 e-6$ \\
1.0 & $7.4091 e-6$ & $7.4091 e-6$ \\
\hline
\end{tabular}

$$
\begin{aligned}
& \odot \widetilde{G}_{k-1}(x, y) d x d y,(F R) \int_{c}^{d}(F R) \\
& \left.\cdot \int_{a}^{b} \mathscr{K}(x, y, s, t) \odot \widetilde{Y}_{k-1}(x, y) d x d y\right), \\
& \leq \epsilon+\mu \int_{c}^{d} \int_{a}^{b}|\mathscr{K}(x, y, s, t)| \\
& \cdot D\left(\widetilde{G}_{k-1}(x, y), \widetilde{Y}_{k-1}(x, y)\right) d x d y .
\end{aligned}
$$

We conclude that

$$
\begin{aligned}
D^{*}\left(\widetilde{G}_{k}, \widetilde{Y}_{k}\right) & \leq \epsilon+\mu \int_{c}^{d} \int_{a}^{b} \mathscr{M} D^{*}\left(\widetilde{G}_{k-1}, \widetilde{Y}_{k-1}\right) d x d y \\
& =\epsilon+B D^{*}\left(\widetilde{G}_{k-1}, \widetilde{Y}_{k-1}\right),
\end{aligned}
$$

and thus

$$
\begin{gathered}
D^{*}\left(\widetilde{G}_{k}, \widetilde{Y}_{k}\right) \leq \epsilon+B D^{*}\left(\widetilde{G}_{k-1}, \widetilde{Y}_{k-1}\right) \\
D^{*}\left(\widetilde{G}_{k-1}, \widetilde{Y}_{k-1}\right) \leq \epsilon+B D^{*}\left(\widetilde{G}_{k-2}, \widetilde{Y}_{k-2}\right) \\
\vdots \vdots \\
D^{*}\left(\widetilde{G}_{1}, \widetilde{Y}_{1}\right) \leq \epsilon+B D^{*}\left(\widetilde{G}_{0}, \widetilde{Y}_{0}\right) .
\end{gathered}
$$

$$
\begin{aligned}
& \leq \epsilon+B \epsilon+B^{2} \epsilon+B^{3} \epsilon+\cdots+B^{k} D^{*}\left(\widetilde{G}_{0}, \widetilde{Y}_{0}\right) \\
& \leq \epsilon\left(1+B+B^{2}+B^{3}+\cdots+B^{k}\right) \leq \frac{\epsilon}{1-B} .
\end{aligned}
$$

Therefore,

$$
\begin{aligned}
& D^{*}\left(\widetilde{u}_{k}, \widetilde{v}_{k}\right) \\
& \quad \leq \frac{1}{1-B}\left(2 m_{l}\left(1+\frac{2 \widetilde{C}}{(d-c)(b-a)}\right)+\epsilon\right) .
\end{aligned}
$$

\section{Numerical Examples}

In this section, we use the proposed method in twodimensional linear fuzzy Fredholm integral equations for solving two examples. By using the proposed method for $n=$ $2, k=5,8,16$, and $r \in\{0.0,0.1,0.2,0.3,0.4,0.5,0.6,0.7,0.8$, $0.9,1.0\}$ in $(s, t)=(0.5,0.5)$, we present the absolute errors in Tables 1-4.

Example 13 (see [11]). Consider the linear integral equation

$$
\begin{aligned}
& \widetilde{G}(s, t) \\
& =\widetilde{g}(s, t) \\
& \quad \oplus(F R) \int_{0}^{1}(F R) \int_{0}^{1} \mathscr{K}(x, y, s, t) \odot \widetilde{G}(x, y) d x d y,
\end{aligned}
$$

with

$$
\mathscr{K}(x, y, s, t)=x y s t
$$

$$
\begin{aligned}
& \underline{\tilde{g}}(s, t, r)=r\left(\frac{1}{3} r+\frac{8}{3}\right)\left(1+s+t-\frac{7}{12} s t\right), \\
& \overline{\widetilde{g}}(s, t, r)=\left(2 r^{2}-4 r+5\right)\left(1+s+t-\frac{7}{12} s t\right) .
\end{aligned}
$$

The exact solution for 2DLFFIE (38) is

$$
\begin{aligned}
& \underline{\widetilde{G}}^{*}(s, t, r)=r\left(\frac{1}{3} r+\frac{8}{3}\right)(s+t+1), \\
& \widetilde{\widetilde{G}}^{*}(s, t, r)=\left(2 r^{2}-4 r+5\right)(s+t+1) .
\end{aligned}
$$


TABLE 2: The absolute errors on the level sets with $n=2, k=16$ for Example 13 by using the proposed method in $(s, t)=(0.5,0.5)$.

\begin{tabular}{lcc}
\hline$r$ level & $\underline{e}^{r}=\left|\underline{\underline{G}}^{*}(s, t, r)-\underline{\underline{u}}_{k}(s, t, r)\right|$ & $\bar{e}^{r}=\left|\overline{\widetilde{G}}^{*}(s, t, r)-\overline{\tilde{u}}_{k}(s, t, r)\right|$ \\
\hline 0.0 & 0. & $1.77636 e-15$ \\
0.1 & $1.11022 e-16$ & $1.77636 e-15$ \\
0.2 & 0. & $1.77636 e-15$ \\
0.3 & $2.22045 e-16$ & $8.88178 e-16$ \\
0.4 & $4.44089 e-16$ & $8.88178 e-16$ \\
0.5 & $4.44089 e-16$ & $8.88178 e-16$ \\
0.6 & $4.44089 e-16$ & 0. \\
0.7 & $8.88178 e-16$ & 0. \\
0.8 & 0. & 0. \\
0.9 & 0. & 0. \\
1.0 & 0. & 0.
\end{tabular}

TABLE 3: The absolute errors on the level sets with $n=2, k=5$ for Example 14 by using the proposed method in $(s, t)=(0.5,0.5)$.

\begin{tabular}{lcc}
\hline$r$ level & $\underline{e}^{r}=\left|\underline{\underline{G}}^{*}(s, t, r)-\underline{\tilde{u}}_{k}(s, t, r)\right|$ & $\bar{e}^{r}=\left|\overline{\widetilde{G}}^{*}(s, t, r)-\overline{\tilde{u}}_{k}(s, t, r)\right|$ \\
\hline 0.0 & 0. & $9.60565 e-13$ \\
0.1 & $4.80276 e-14$ & $9.12548 e-13$ \\
0.2 & $9.60551 e-14$ & $8.64531 e-13$ \\
0.3 & $1.44079 e-13$ & $8.16458 e-13$ \\
0.4 & $1.9211 e-13$ & $7.68441 e-13$ \\
0.5 & $2.40141 e-13$ & $7.20479 e-13$ \\
0.6 & $2.88158 e-13$ & $6.72407 e-13$ \\
0.7 & $3.36203 e-13$ & $6.24334 e-13$ \\
0.8 & $3.8422 e-13$ & $5.76317 e-13$ \\
0.9 & $4.32265 e-13$ & $5.283 e-13$ \\
1.0 & $4.80282 e-13$ & $4.80282 e-13$ \\
\hline
\end{tabular}

TABLE 4: The absolute errors on the level sets with $n=2, k=8$ for Example 14 by using the proposed method in $(s, t)=(0.5,0.5)$.

\begin{tabular}{|c|c|c|}
\hline$r$-level & $\underline{e}^{r}=\left|\widetilde{G}^{*}(s, t, r)-\underline{\tilde{u}}_{k}(s, t, r)\right|$ & $\bar{e}^{r}=\mid \overline{\widetilde{G}}^{*}(s, t, r)-\overline{\widetilde{u}}_{k}(s, t, r)$ \\
\hline 0.0 & 0. & 0. \\
\hline 0.1 & 0. & 0. \\
\hline 0.2 & 0. & $5.55112 e-17$ \\
\hline 0.3 & 0. & 0 \\
\hline 0.4 & 0. & 0 \\
\hline 0.5 & 0. & $5.55112 e-17$ \\
\hline 0.6 & 0. & 0 \\
\hline 0.7 & 0. & 0. \\
\hline 0.8 & 0. & 0. \\
\hline 0.9 & $2.77556 e-17$ & 0. \\
\hline 1.0 & 0. & 0. \\
\hline
\end{tabular}

Example 14 (see [13]). Consider the following fuzzy Fredholm integral equation (38) with

$$
\begin{aligned}
& \underline{\tilde{g}}(s, t, r)=r\left(s t+\frac{1}{676}\left(s^{2}+t^{2}-2\right)\right), \\
& \overline{\widetilde{g}}(s, t, r)=(2-r)\left(s t+\frac{1}{676}\left(s^{2}+t^{2}-2\right)\right),
\end{aligned}
$$

and kernel

$$
\begin{aligned}
& \mathscr{K}(x, y, s, t)=\frac{1}{169}\left(x^{2}+y^{2}-2\right)\left(s^{2}+t^{2}-2\right), \\
& 0 \leq s, t, x, y \leq 1 .
\end{aligned}
$$

The exact solution is

$$
\underline{\widetilde{G}}^{*}(s, t, r)=r s t,
$$




$$
\overline{\widetilde{G}}^{*}(s, t, r)=(2-r) s t .
$$

\section{Conclusion}

The 2DLFFIE is solved by utilizing two-dimensional fuzzy Lagrange interpolation and iterative method. As it was expected the method used to approximate the integral in this equation is a suitable one since convergence analysis and stability analysis have been proved and also absolute error in examples is nearly zero. As a result, considering the fact that the proposed method does not lead to solve fuzzy linear system, it can be utilized as an efficient method to solve this type of equations. As future researches, we can use finite and divided differences methods, fuzzy spline interpolation, and fuzzy quasi-interpolation for solving two-dimensional linear or nonlinear fuzzy Fredholm integral equations.

\section{Conflicts of Interest}

The authors declare that there are no conflicts of interest related to this paper.

\section{References}

[1] E. Babolian, H. Sadeghi Goghary, and S. Abbasbandy, "Numerical solution of linear Fredholm fuzzy integral equations of the second kind by Adomian method," Applied Mathematics and Computation, vol. 161, no. 3, pp. 733-744, 2005.

[2] A. M. Bica, "Error estimation in the approximation of the solution of nonlinear fuzzy Fredholm integral equations," Information Sciences, vol. 178, no. 5, pp. 1279-1292, 2008.

[3] A. M. Bica and C. Popescu, "Approximating the solution of nonlinear Hammerstein fuzzy integral equations," Fuzzy Sets and Systems, vol. 245, pp. 1-17, 2014.

[4] M. A. Fariborzi Araghi and G. Kazemi Gelian, "Solving fuzzy Fredholm linear integral equations using Sinc method and double exponential transformation," Soft Computing, vol. 19, no. 4, pp. 1063-1070, 2015.

[5] M. A. Faborzi Araghi and N. Parandin, "Numerical solution of fuzzy Fredholm integral equations by the Lagrange interpolation based on the extension principle," Soft Computing, vol. 15, no. 12, pp. 2449-2456, 2011.

[6] H. Hosseini Fadravi, R. Buzhabadi, and H. Saberi Nik, "Solving linear Fredholm fuzzy integral equations of the second kind by artificial neural networks," Alexandria Engineering Journal, vol. 53, no. 1, pp. 249-257, 2014.

[7] Y. Jafarzadeh, "Numerical solution for fuzzy Fredholm integral equations with upper-bound on error by splines interpolation," Fuzzy Information and Engineering, vol. 4, no. 3, pp. 339-347, 2012.

[8] A. Molabahrami, A. Shidfar, and A. Ghyasi, "An analytical method for solving linear Fredholm fuzzy integral equations of the second kind," Computers \& Mathematics with Applications, vol. 61, no. 9, pp. 2754-2761, 2011.

[9] N. Parandin and M. A. Fariborzi Araghi, "The approximate solution of linear fuzzy Fredholm integral equations of the second kind by using iterative interpolation," World Academy of Science, Engineering and Technology, vol. 49, pp. 978-984, 2009.
[10] A. M. Bica and S. Ziari, "Iterative numerical method for fuzzy Volterra linear integral equations in two dimensions," Soft Computing, vol. 21, no. 5, pp. 1097-1108, 2017.

[11] S. M. Sadatrasoul and R. Ezzati, "Quadrature Rules and Iterative Method for Numerical Solution of Two-Dimensional Fuzzy Integral Equations," Abstract and Applied Analysis, vol. 2014, Article ID 413570, 18 pages, 2014.

[12] S. M. Sadatrasoul and R. Ezzati, "Iterative method for numerical solution of two-dimensional nonlinear fuzzy integral equations," Fuzzy Sets and Systems, vol. 280, pp. 91-106, 2015.

[13] F. Mirzaee, M. K. Yari, and E. Hadadiyan, "Numerical solution of two-dimensional fuzzy Fredholm integral equations of the second kind using triangular functions," Beni-Suef University Journal of Basic and Applied Sciences, vol. 4, no. 2, pp. 109-118, 2015.

[14] H. Nouriani and R. Ezzati, "Quadrature iterative method for numerical solution of two-dimensional linear fuzzy Fredholm integral equations," Mathematical Sciences, vol. 11, no. 1, pp. 6372, 2017.

[15] R. Ezzati and S. Ziari, "Numerical solution of two-dimensional fuzzy Fredholm integral equations of the second kind using fuzzy bivariate Bernstein polynomials," International Journal of Fuzzy Systems, vol. 15, no. 1, pp. 84-89, 2013.

[16] N. Hassasi and R. Ezzati, "Numerical solution of twodimensional fuzzy Fredholm integral equations using collocation fuzzy wavelet like operator," International Journal of Industrial Mathematics, vol. 7, p. 11, 2015.

[17] M. A. Vali, M. J. Agheli, and S. Gohari Nezhad, "Homotopy analysis method to solve two-dimensional fuzzy Fredholm integral equation," General Mathematics Notes, vol. 22, pp. 3143, 2014.

[18] A. M. Bica and S. Ziari, "Open fuzzy cubature rule with application to nonlinear fuzzy Volterra integral equations in two dimensions," Fuzzy Sets and Systems, In press, Corrected Proof.

[19] A. Rivaz and F. Yousefi, "Kernel iterative method for solving two-dimensional fuzzy Fredholm integral equations of the second kind," Journal of Fuzzy Set Valued Analysis, vol. 2013, p. 9, 2013.

[20] A. Rivaz and F. Yousefi, "Modified homotopy perturbation method for solving two-dimensional fuzzy Fredholm integral equation," International Journal of Applied Mathematics, vol. 25, no. 4, pp. 591-602, 2012.

[21] A. Rivaz, F. Yousefi, and H. Salehinejad, "Using block pulse functions for solving two-dimensional fuzzy Fredholm integral equations of the second kind," International Journal of Applied Mathematics, vol. 25, no. 4, pp. 571-582, 2012.

[22] S. M. Sadatrasoul and R. Ezzati, "Numerical solution of twodimensional nonlinear Hammerstein fuzzy integral equations based on optimal fuzzy quadrature formula," Journal of Computational and Applied Mathematics, vol. 292, pp. 430-446, 2016.

[23] S. Ziari, "Iterative method for solving two-dimensional nonlinear fuzzy integral equations using fuzzy bivariate blockpulse functions with error estimation," Iranian Journal of Fuzzy Systems, vol. 15, no. 1, pp. 55-76, 183, 2018.

[24] G. A. Anastassiou, Fuzzy Mathematics: Approximation Theory, vol. 251, Springer, 2010.

[25] A. R. Bozorgmanesh, M. Otadi, A. A. Safe Kordi et al., "Barkhordari Ahmadi, Lagrange two-dimensional interpolation method for modeling nanoparticle formation during RESS process," International Journal of Industrial Mathematics, vol. 1, pp. 175181, 2009. 


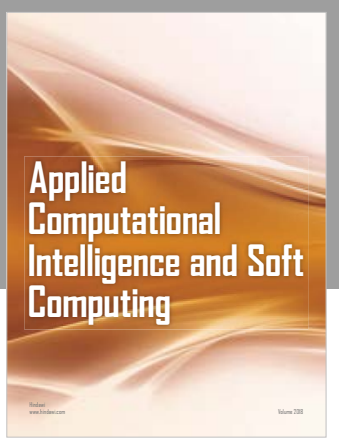

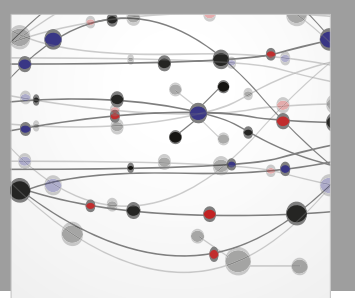

The Scientific World Journal
Submit your manuscripts at

Computing
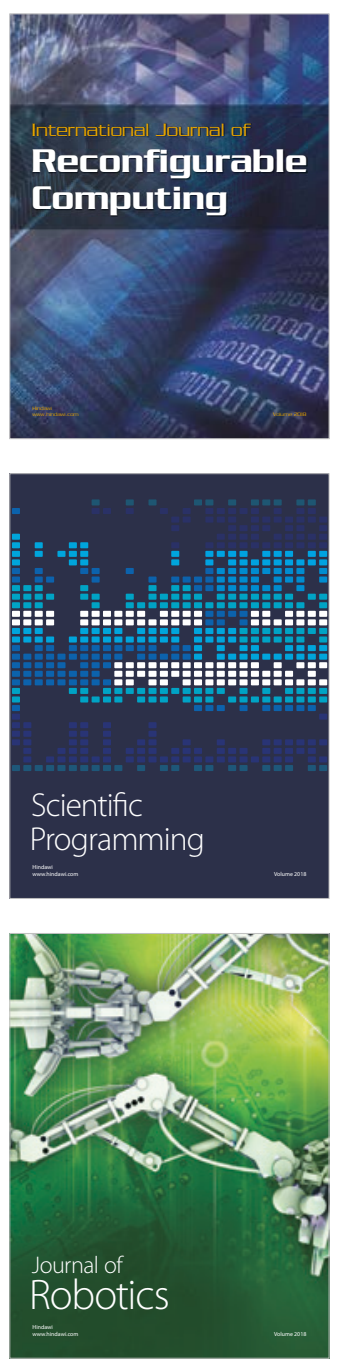

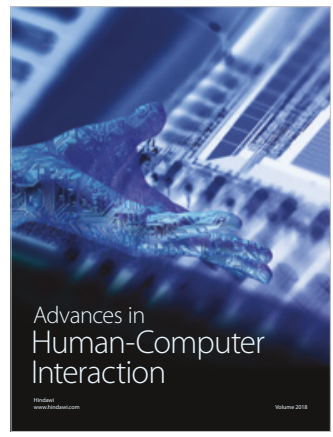

Human-Compute

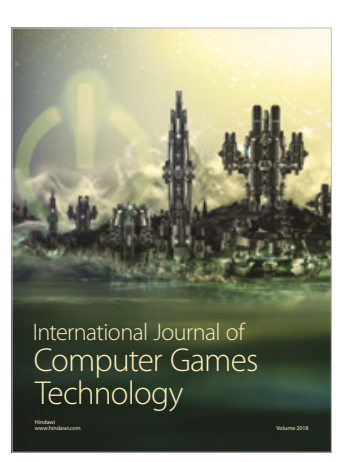

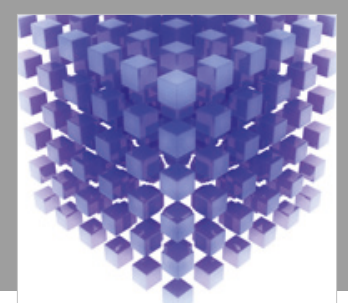

Mathematical Problems in Engineering

\section{Engincering}
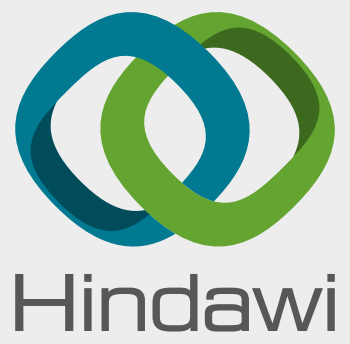

www.hindawi.com
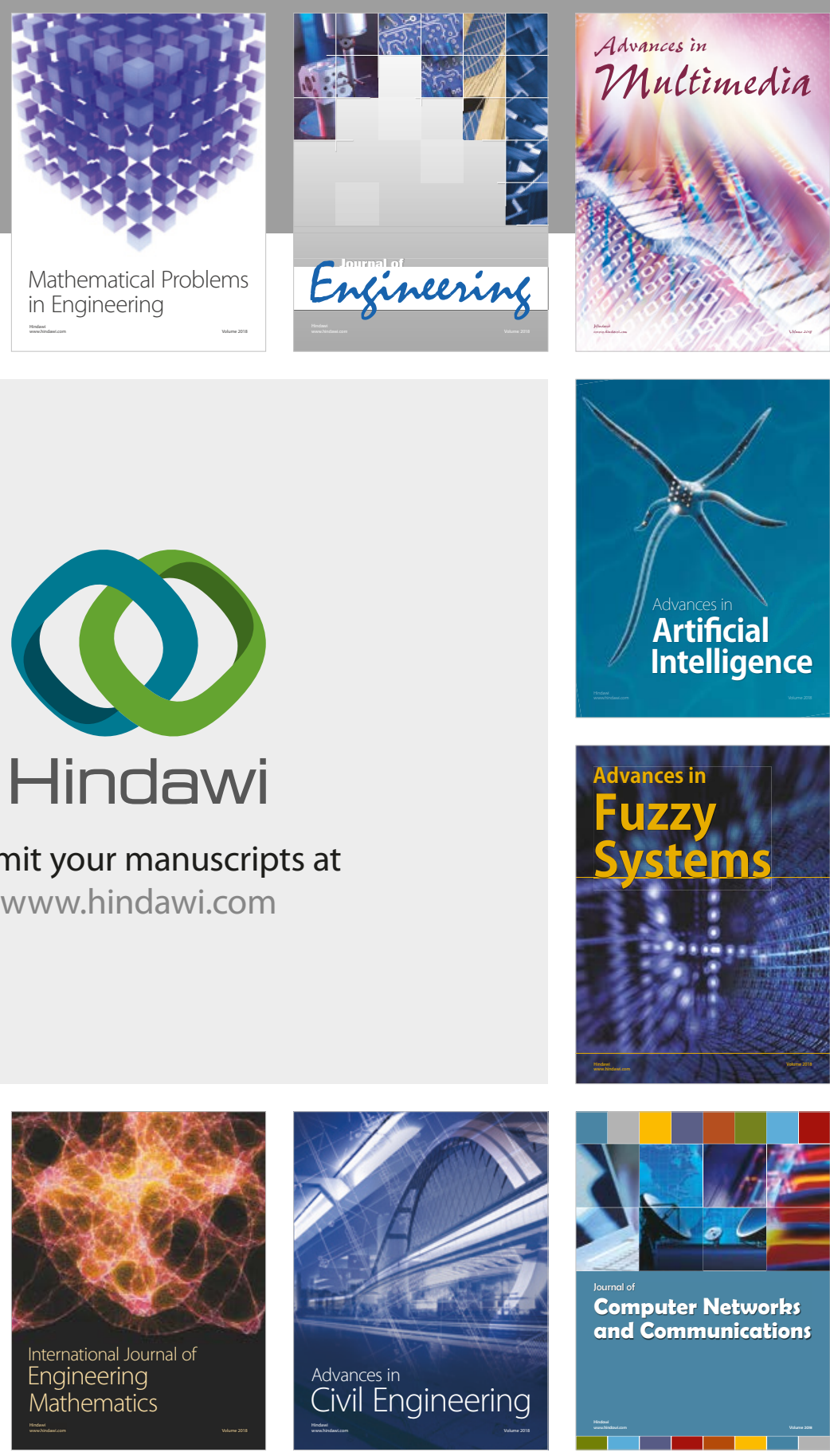

Computer Networks and Communications

Multimedia
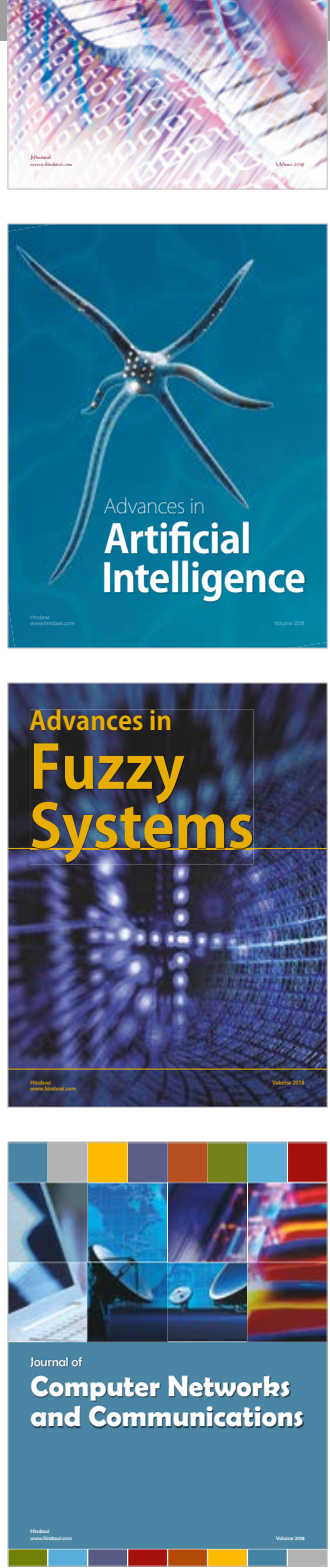

Advances in

Modelling \&

Simulation

in Engineering

interaction

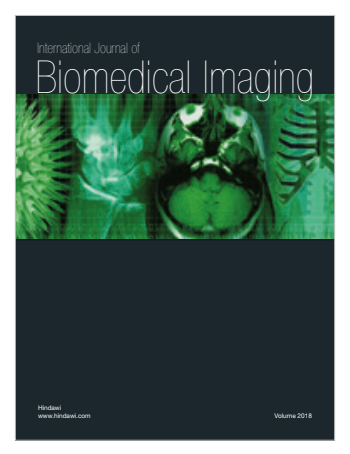

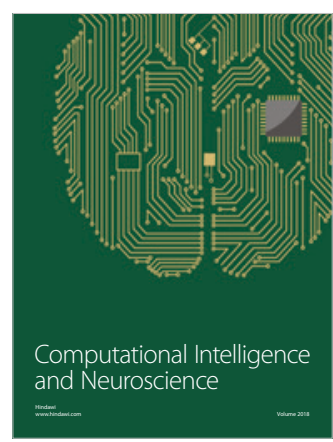

